\title{
ANALYSIS OF NONLINEAR GYROMAGNETIC LINE OPERATION USING LLG EQUATION *
}

\author{
J.O. Rossi ${ }^{\xi}$, F.S. Yamasaki, E. Schamiloglu ${ }^{+}$,J.J. Barroso ${ }^{++}$ \\ National Institute for Space Research, Plasma Laboratory, PO Box 12245-970 \\ Sao Jose dos Campos, SP, Brazil
}

\section{Abstract}

This paper deals with the study of the gyromagnetic effect since it has proven to be an excellent way of generating radiofrequency (RF) using ferrite loaded nonlinear lines in the range of $\mathrm{GHz}$. Using a proper mathematical model based on the analysis of the LLG (Landau-Lifshitz-Gilbert) equation will be demonstrated the dependence of the center frequency generated on both the intensity of the axial magnetic applied and the incident pulse amplitude.

\section{INTRODUCTION}

Nowadays gyromagnetic nonlinear transmission lines (NLTL) have been studied with great interest [1] since they can generate RF up to frequencies of $1-3 \mathrm{GHz}$ at high power (hundreds of MW) as demonstrated recently [2-4]. As they are all solid-sate they can be used as compact RF sources. In these devices microwaves are induced by the damped gyromagnetic precession of the magnetic moments in the ferrimagnetic material as their coaxial structure are loaded with ferrite material as magnetic medium. As observed, the operational performance of gyromagnetic NLTLs strongly depend on the amplitude of the incident pulse and on the static axial magnetic bias. In fact, this phenomenon can be predicted using LLG equation, which provides that the precession frequency is directly proportional to the total effective magnetic field, composed by the sum of the axial field plus the azimuthal field generated by the incident pulse current into the line $\left(\omega=\gamma H_{\mathrm{t}}\right)$. The LLG equation represents the dynamic process of the magnetization $M$ inside the line ferrite, consisting of the precession (first term) followed by the damping movement (second term), which is in the form

$$
\frac{\partial \vec{M}}{\partial t}=\gamma \mu_{0} \vec{M} \times \overrightarrow{H_{t}}-\frac{\alpha}{M_{s}} \vec{M} \times \frac{\partial \vec{M}}{\partial t},
$$

where $H_{t}$ is the total effective magnetic field, $\gamma=1.76 \times 10^{11}$ $\mathrm{rad} / \mathrm{s} / \mathrm{T}$ the electron gyromagnetic ratio, $\mu_{0}=4 \pi \times 10^{-7} \mathrm{H} / \mathrm{m}$ the free space magnetic permeability, and $M s$ the medium saturated static magnetization (generally assumed $0.35 \mathrm{~T}$ for ferrites).

However, as shown in [2-4] the NLTL performance does not confirm this result. As not expected, and different from/contrary to what the LLG equation predicts, the experimental trend observed indicates that the center frequency decreases with the static axial magnetic, but increases with the incident input pulse amplitude because of the azimuthal field. A possible explanation for this is that the TEM mode wave propagates down the coaxial line coupled to the azimuthal magnetic field. Thus, the objective of this paper is to address this problem correctly by doing a mathematical analysis using the LLG equation for the TEM mode propagation and neglecting the damping term. With this proper formulation, it will be demonstrated the experimental frequency dependence observed for the gyromagnetic NLTL.

\section{PROPOSED MODEL}

First, it is assumed a plane wave propagating in an infinite ferrite medium in the plane $x-y$ under an intense axial magnetic bias $\mathbf{H}$ in the $\mathbf{z}$-direction bias as illustrated in Fig. 1. If the ferrite goes into saturation upon application of the axial bias, the medium becomes anisotropic and the AC applied field $\mathbf{h}$ in the plane $\mathrm{x}-\mathrm{y}$ is not aligned with its corresponding magnetization $\mathbf{m}$ as shown in Fig. 1. Let $\mathbf{M}_{\mathbf{s}}$ be the corresponding static magnetization in the axial or z-direction generated by the field $\mathbf{H}$, the total $\mathbf{M}$ magnetization using the LLG equation and neglecting its dissipative term is given by

$$
\frac{\partial \vec{M}}{\partial t}=\mu_{0} \gamma \vec{M} \times \vec{H}_{t}=\mu_{0} \gamma\left(\vec{M}_{s}+\vec{m}\right) \times(\vec{H}+\vec{h}) .
$$

\footnotetext{
${ }^{*}$ Work supported in part by SOARD-USAF under contract number FA9550-14-1-0133

$\xi$ email: jose.rossi@inpe.br

${ }^{+}$University of New Mexico, Electrical and Computer Eng. Department, Albuquerque, NM, USA

${ }^{+}$Technological Institute of Aeronautics, Division of Electronic Eng., Sao Jose dos Campos, SO, Brazil
} 


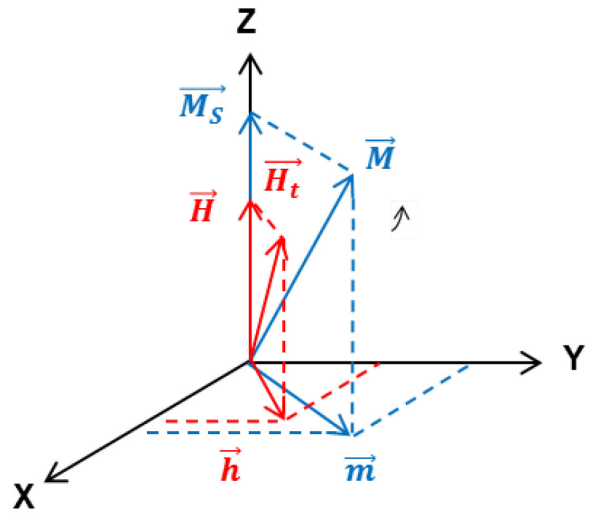

Figure 1. The rotating total magnetization $M$ around $z$ caused by the AC field $\mathbf{h}$ applied.

Since $\vec{M}_{s} \times \vec{H}=0$ and neglecting the second small term $\vec{m} \times \vec{h}$ if $\vec{h} \ll \vec{H}$ and $\vec{m} \ll \vec{M}$ then (2) becomes

$$
\frac{\partial \vec{M}}{\partial t}=\mu_{0} \gamma M_{s} \hat{z} \times \vec{h}-\mu_{0} \gamma H \hat{z} \times \vec{m} .
$$

Separating the components of the fields in coordinates $\mathrm{x}, \mathrm{y}$ and $\mathrm{z}$ gives

$$
\begin{aligned}
& \frac{d m_{x}}{d t}=-\omega_{m} h_{y}+\omega_{0} m_{y} \\
& \frac{d m_{y}}{d t}=\omega_{m} h_{x}-\omega_{0} m_{x} \\
& \frac{d m_{z}}{d t}=0
\end{aligned}
$$

where $\omega_{m}=\mu_{0} \gamma M_{s}$ and $\omega_{0}=\mu_{0} \gamma H$. Using the tensor form for the magnetization $\mathbf{m}$ as a function of the magnetic susceptibilities in $\mathrm{x}, \mathrm{y}$, and $\mathrm{z}$ directions gives

$$
\vec{m}=\left[\begin{array}{l}
m_{x} \\
m_{y} \\
m_{z}
\end{array}\right]=\left[\begin{array}{ccc}
\chi_{x x} & \chi_{x y} & 0 \\
\chi_{y x} & \chi_{y y} & 0 \\
0 & 0 & 0
\end{array}\right]\left[\begin{array}{c}
h_{x} \\
h_{y} \\
0
\end{array}\right]=[\chi](\vec{h})
$$

Thus, (4) and (5) become:

$$
\begin{gathered}
\frac{d m_{x}}{d t}=-\omega_{m} \frac{m_{y}}{\chi_{y y}}+\omega_{0} m_{y}=-\left(\frac{\omega_{m}}{\chi_{y y}}-\omega_{0}\right) m_{y} \\
\frac{d m_{y}}{d t}=\omega_{m} \frac{m_{x}}{\chi_{x x}}-\omega_{0} m_{x}=\left(\frac{\omega_{m}}{\chi_{x x}}-\omega_{0}\right) m_{x}
\end{gathered}
$$

where in this case $\chi_{x x}=\chi_{y y}=\chi$ and $\chi_{x y}=\chi_{y x}=0$ required by the mode symmetry as gyromagnetic lines operate in the TEM mode and the AC magnetic field sees the ferrite as a uniaxial material. By taking the second derivative of (8) and using in (9) or vice-versa, they can be reduced to the set of equations for the harmonic oscillator such as

$$
\begin{aligned}
& \frac{d^{2} m_{x}}{d t^{2}}+\left(\frac{\omega_{m}}{\chi}-\omega_{0}\right)^{2} m_{x}=0 \\
& \frac{d^{2} m_{y}}{d t^{2}}+\left(\frac{\omega_{m}}{x}-\omega_{0}\right)^{2} m_{y}=0
\end{aligned}
$$

with the precession angular frequency $\omega_{p}$ given by

$$
\omega_{p}=\frac{\omega_{m}}{\chi}-\omega_{0}
$$

From (12) the corresponding precession frequency is

$$
f=\frac{\gamma}{2 \pi}\left(\frac{\mu_{0}}{\chi} M_{s}-\mu_{0} H\right)
$$

where the constant $\gamma / 2 \pi \cong 28 \mathrm{GHz} / \mathrm{T}, \mu_{0} M_{s}=0.35 \mathrm{~T}$ is the saturated ferrite magnetization in z-direction, $H$ is the bias axial field in $\mathrm{A} / \mathrm{m}$ in z-direction, and $\chi$ is the azimuthal magnetic susceptibility.

\section{DISCUSSION}

Fig. 2 illustrates how the proposed model works for a variation of the bias axial field between 5 and $45 \mathrm{kA} / \mathrm{m}$. As the bias field increases the precession frequency decreases following a linear decay. The linear decay slope is constant and its value does not depend on the azimuthal magnetic susceptibility, but higher frequencies can be achieved with lower $\chi$. This can be reached by applying incident pulses of higher amplitudes into the NLTL since higher pulse current will produce more intense azimuthal field, which in turn tend to saturate the magnetic medium (ferrite) decreasing $\chi$.

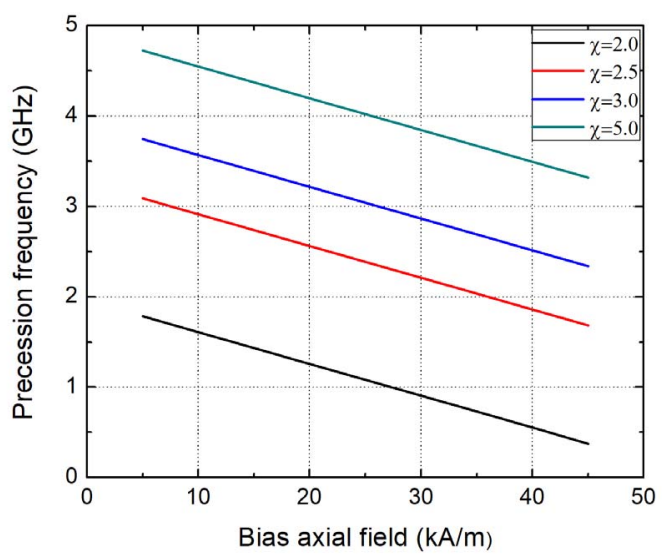

Figure 2. NLTL frequency varying the static axial magnetic field for different values of $\chi$.

In the literature, it is possible to find several experiments with gyromagnetic oscillators whose results 
obtained are consistent with the proposed model. For instance, Bragg et al. [2] used a ferrite loaded NiZn NLTL of the order of $1 \mathrm{~m}$ length or less with magnetic axial bias varying between $10-40 \mathrm{kA} / / \mathrm{m}$ approximately and driven by exciting input pulse voltages between $20-30 \mathrm{kV}$. They obtained a center frequency variation between 2.7 and 1.7 $\mathrm{GHz}$ when the bias axial field was increased from 10 to $40 \mathrm{kA} / \mathrm{m}$ for an incident pulse amplitude of $20 \mathrm{kV}$. Increasing the amplitude of the incident pulse up to 25 and $30 \mathrm{kV}$ provided frequency variations in the range of 3.1-2.0 GHz and 3.5-2.2 GHz, respectively, as $\chi$ drops at higher voltages, which is in accordance with the model. Another example is found in Karelin et al. [3] where they tested a NiZn NLTL with a $38 \Omega$ load and an incident pulse amplitude of $100 \mathrm{kV}$. Experimentally they produced a center frequency varying between 1.4 and $1.0 \mathrm{GHz}$ for a corresponding axial field increase from 20 to $80 \mathrm{kA} / \mathrm{m}$ approximately, confirming again the inverse dependence of frequency with $\mathrm{H}$ as proposed by the present model. Finally, Romanchenko et al. [4] observed the same effect using a $700 \mathrm{~mm}$ four-channel NTTL of NiZn ferrites coupled to four helix antennas. In this case, they observed frequency decrease from 1.7 and $1.2 \mathrm{GHz}$ for a corresponding $\mathrm{H}$-field increase from 15 to $40 \mathrm{kA} / \mathrm{m}$ approximately for incident pulse amplitudes above 200 $\mathrm{kV}$.

\section{IV.SUMMARY}

In this paper, an analytic model is proposed to predict the center frequency in NLTLs, which decreases as the bias axial filed is increased. The model is based on the analysis of LLG by neglecting the dissipative term. The model behavior was checked observing the experimental data found in the literature for several gyromagnetic oscillators, which confirmed the consistency between model and experiments. The main point herein is that our proposed model assumes TEM mode propagation inside the ferrites (azimuthal symmetry). This is an important issue as earlier models used in numerical simulations did not take this fact into consideration.

As future work, the incorporation of the TL telegraphist equations into the model has been considered to study the conditions on wave propagation mode along the line without attenuation.

\section{V.REFERENCES}

[1] F.S. Yamasaki, E. Schamiloglu, J.O. Rossi, J.J. Barroso, "Simulation studies of distributed nonlinear gyromagnetic lines based on LC lumped model". IEEE Trans. Plasma Sci. 44 (10), pp. 2232-2239, 2016.

[2] J-W. B. Bragg, J.C. Dickens, and A.A. Neuber, "Material selection considerations for coaxial, ferrimagnetic-based nonlinear transmission lines," Journal of Applied Physics 113, 064904 (2013).

[3] S. Y. Karelin, V. B. Krasovitsky, I. I. Magda, V. S. Mukhin, and V. G. Sinitsin, RF Oscillations in a Coaxial Transmission Line with a Saturated Ferrite: 2-D Simulation and Experiment, 2016 8th International Conference on Ultrawideband and Ultrashort Impulse Signals, 5-11 Sept. 2016, Odessa, Ukraine, pp. 60-63.

[4] I. V. Romanchenko, V. V. Rostov, A. V. Gunin, and V. Yu. Konev,"High power microwave beam steering based on gyromagnetic nonlinear transmission lines," Journal of Applied Physics 117, 214907 (2015). 